INPLASY

PROTOCOL

To cite: Chen et al. Effects of Baduanjin on postoperative rehabilitation of patients with breast cancer: A protocol for systematic review and metaanalysis. Inplasy protocol

202140018. doi:

10.37766/inplasy2021.4.0018

Received: 03 April 2021

Published: 04 April 2021

Corresponding author:

Bin Pu

287147472@qq.com

Author Affiliation:

Traditional medical diagnosis and treatment centre, Gansu Provincial People's Hospital, Lanzhou, China

Support: Gansu University of TCM.

Review Stage at time of this submission: The review has not yet started.

Conflicts of interest:

None declared.

\section{Effects of Baduanjin on postoperative rehabilitation of patients with breast cancer: A protocol for systematic review and meta-analysis}

Chen, G1; Lin, Y2; Zhao, X33 Pu, B4.

Review question / Objective: To evaluate the effects of Baduanjin on postoperative rehabilitation of patients with breast cancer.

Condition being studied: Breast cancer patients over 18 years old.

Information sources: Experienced researchers will develop a comprehensive search strategy. The literature search will be conducted on the following database: PubMed, Embase. com, the Cochrane Central Register of Controlled Trials (CENTRAL), Web of Science, China National Knowledge Infrastructure (CNKI), Wan Fang, and SinoMed. Retrieve time is from the establishment of the database to June 1, 2021. We will also manually search the references of related articles.

INPLASY registration number: This protocol was registered with the International Platform of Registered Systematic Review and Meta-Analysis Protocols (INPLASY) on 04 April 2021 and was last updated on 04 April 2021 (registration number INPLASY202140018).

\section{INTRODUCTION}

Review question / Objective: To evaluate the effects of Baduanjin on postoperative rehabilitation of patients with breast cancer.
Condition being studied: Breast cancer patients over 18 years old.

\section{METHODS}

Search strategy: \#1 "Breast Neoplasms"[Mesh] OR "Breast Carcinoma 
In Situ"[Mesh] OR "Breast Neoplasms, Male"[Mesh] OR "Carcinoma, Ductal, Breast"[Mesh] OR "Carcinoma, Lobular"[Mesh] OR "Inflammatory Breast Neoplasms"[Mesh] OR "Triple Negative Breast Neoplasms"[Mesh] OR "Unilateral Breast Neoplasms"[Mesh] OR breast neoplasm*[Title/Abstract] OR breast tumor*[Title/Abstract] OR breast carcinoma*[Title/Abstract] OR breast cancer*[Title/Abstract] OR breast tumour*[Title/Abstract] OR mammary neoplasm*[Title/Abstract] OR mammary tumor*[Title/Abstract] OR mammary carcinoma*[Title/Abstract] OR mammary cancer*[Title/Abstract] OR mammary tumour*[Title/Abstract] OR breast adenocarcinoma*[Title/Abstract] OR breast carcinogenesis[Title/Abstract] OR breast sarcoma*[Title/Abstract] OR phyllodes tumor*[Title/Abstract] OR intraductal carcinoma*[Title/Abstract] OR lobular carcinoma* [Title/Abstract]; \# 2 Baduanjin[Title/Abstract] OR Baduan jin[Title/Abstract] OR Baduanjin exercise*[Title/Abstract] OR BDJ[Title/ Abstract] OR eight section brocade*[Title/ Abstract] OR eight-section Brocade* [Title/ Abstract] OR eight trigrams boxing[Title/ Abstract] OR eight-treasured exercise*[Title/Abstract] OR eight pieces of brocade*[Title/Abstract] OR eight brocade section[Title/Abstract] OR Chinese regimen*[Title/Abstract] OR Chinese ancient regimen*[Title/Abstract] OR "qigong"[Mesh] OR qigong[Title/Abstract] OR Qi Gong[Title/Abstract] OR Ch'iKung*[Title/Abstract] OR "rehabilitation exercise"[Mesh] OR rehabilitation exercise*[Title/Abstract] OR Remedial Exercise $^{\star}[$ Title/Abstract] OR Exercise, Remedial*[Title/Abstract] OR Exercises, Remedial*[Title/Abstract] OR Remedial Exercises*[Title/Abstract] OR Therapy, Exercise*[Title/Abstract] OR Exercise Therapies*[Title/Abstract] OR Therapies, Exercise ${ }^{\star}[$ Title/Abstract] OR Rehabilitation Exercise*[Title/Abstract] OR Exercise, Rehabilitation [Title/Abstract] OR Exercises, Rehabilitation*[Title/Abstract] OR Rehabilitation Exercises*[Title/ Abstract]; \#3 "Clinical Trials, Phase II as Topic"[Mesh] OR "Clinical Trials, Phase III as Topic"[Mesh] OR "Clinical Trials, Phase
IV as Topic"[Mesh] OR "Controlled Clinical Trials as Topic"[Mesh] OR "Randomized Controlled Trials as Topic"[Mesh] OR "Intention to Treat Analysis"[Mesh] OR "Pragmatic Clinical Trials as Topic"[Mesh] OR "Clinical Trials, Phase II"[Publication Type] OR "Clinical Trials, Phase III"[Publication Type] OR "Clinical Trials, Phase IV"[Publication Type] OR "Controlled Clinical Trials"[Publication Type] OR "Randomized Controlled Trials"[Publication Type] OR "Pragmatic Clinical Trials as Topic"[Publication Type] OR "Single-Blind Method"[Mesh] OR "Double-Blind Method"[Mesh] OR random*[Title/Abstract] OR blind*[Title/Abstract] OR singleblind* [Title/Abstract] OR doubleblind*[Title/Abstract] OR trebleblind * [Title/Abstract] OR tripleblind*[Title/Abstract]; 4 \#1 AND \#2 AND \#3.

Participant or population: Breast cancer patients over 18 years old confirmed by pathology or by cytology received treatment, such as surgery, chemotherapy, radiotherapy, and hormone therapy, regardless of region, race, and education.

Intervention: Intervention is that using Baduanjin in rehabilitation, whether or not combined with routine drug therapy. There are no restrictions on the type, frequency, length, and stage.

Comparator: The control group can be any other treatments, such as physical training in other forms, conventional nursing measures, psychological therapy, routine activities, and no treatment.

Study designs to be included: Studies must be randomized controlled trials (RCTs) that assessed the effects of Baduanjin on breast cancer patients. Reviews, conference proceedings, case reports, observational studies, non-random studies will be excluded.

Eligibility criteria: The effects of Baduanjin on postoperative rehabilitation of patients with breast cancer. 
Information sources: Experienced researchers will develop a comprehensive search strategy. The literature search will be conducted on the following database: PubMed, Embase. com, the Cochrane Central Register of Controlled Trials (CENTRAL), Web of Science, China National Knowledge Infrastructure (CNKI), Wan Fang, and SinoMed. Retrieve time is from the establishment of the database to June 1, 2021. We will also manually search the references of related articles.

Main outcome(s): The outcomes can be quality of postoperation life, emotions (depression, anxiety), or physical rehabilitation (body mass index, heart rate variability, lung capacity, arm circumference, shoulder range of motion, step test index).

Data management: Two independent researchers will extract data according to the following items: author, year, funding, number of patients, publication time, study period, grouping and sample size, age and sex of patients, intervention method, basic treatments, whether to adopt the blindness, the primary and secondary outcome, and follow-up time. We will utilize the formal data extract sheets in Excel to execute the program. When these items arise disagreement, we will solve it through the third reviewer.

Quality assessment / Risk of bias analysis: The risk of bias assessment will be implemented by two trained reviewers independently. We will use the "Cochrane bias risk assessment tool". The assessment list including the following seven items:(1) generation of random sequence (selection bias). (2) allocation concealment (selection bias). (3) blinding of participants and researchers (performance bias). (4) blinding of outcome assessment (detection bias). (5) integrity outcome data (attrition bias). (6) selective reporting (reporting bias). (7) other sources of bias (other bias) Each item will be classified as low risk, high risk, and unclear risk. Two experienced researchers will accomplish this job. Contradictory will reach agreements through negotiation or by the third researcher.

Strategy of data synthesis: Statistical analysis will be performed with software Stata (13.0; Stata Corporation, College Station, TX). We will conduct metaanalyses using the inverse variance method to compute relative risks (RRs) and their $95 \%$ confidence interval (CI) for dichotomous outcomes and mean difference (MD) and $95 \% \mathrm{Cl}$ for continuous outcomes. The statistical level of significance will be set at $P<0.05$.

Subgroup analysis: While heterogeneity exists among studies, we will utilize subgroup analysis or sensitivity analysis. Subgroup analysis will be conducted on different sectionalization (age, operational styles, outcomes, whether Baduanjin unites with regular medical therapy) to explore the source of heterogeneity.

Sensitivity analysis: Sensitivity analysis, differing from the subgroup analysis, can locate the low-quality studies in metaanalysis. The analysis will replicate many times by eliminating one study each time. For each analysis, we will get an overall effect value and $95 \% \mathrm{Cl}$. If the data changes little, we can confirm that the result of the.

Country(ies) involved: China.

Keywords: Baduanjin, Effect, Breast cancer, Rehabilitation, Systematic review.

Contributions of each author:

Author 1 - Guangyan Chen.

Email: 495466026@qq.com

Author 2 - Yuqin Lin.

Email: 1026266349@qq.com

Author 3 - Xiyun Zhao.

Email: 1071386436@qq.com

Author 4 - Bin Pu.

Email: 287147472@qq.com 